\title{
Canadian Thoracic Society 2012 guideline update: Diagnosis and management of asthma in preschoolers, children and adults: Executive summary
}

\author{
M Diane Lougheed MD MSc${ }^{1}$, Catherine Lemiere MD², Francine M Ducharme MD MSc ${ }^{2}$, Chris Licskai MD³, \\ Sharon D Dell MD', Brian H Rowe MD MSc${ }^{5}$, Mark FitzGerald $\mathrm{MD}^{6}$, Richard Leigh $\mathrm{MD} \mathrm{PhD}^{7}$, Wade Watson $\mathrm{MD}^{8}$, \\ Louis-Philippe Boulet MD ${ }^{9}$; Canadian Thoracic Society Asthma Clinical Assembly
}

\begin{abstract}
MD Lougheed, C Lemiere, FM Ducharme, et al; Canadian Thoracic Society Asthma Clinical Assembly. Canadian Thoracic Society 2012 guideline update. Diagnosis and management of asthma in preschoolers, children and adults: Executive summary. Can Respir J 2012;19(6):e81-e88.
\end{abstract}

BACKGROUND: In 2010, the Canadian Thoracic Society (CTS) published a Consensus Summary for the diagnosis and management of asthma in children six years of age and older, and adults, including an updated Asthma Management Continuum. The CTS Asthma Clinical Assembly subsequently began a formal clinical practice guideline update process, focusing, in this first iteration, on topics of controversy and/or gaps in the previous guidelines.

METHODS: Four clinical questions were identified as a focus for the updated guideline: the role of noninvasive measurements of airway inflammation for the adjustment of anti-inflammatory therapy; the initiation of adjunct therapy to inhaled corticosteroids (ICS) for uncontrolled asthma; the role of a single inhaler of an ICS/long-acting beta ${ }_{2}$-agonist combination as a reliever, and as a reliever and a controller; and the escalation of controller medication for acute loss of asthma control as part of a self-management action plan. The expert panel followed an adaptation process to identify and appraise existing guidelines on the specified topics. In addition, literature searches were performed to identify relevant systematic reviews and randomized controlled trials. The panel formally assessed and graded the evidence, and made 34 recommendations.

RESULTS: The updated guideline recommendations outline a role for inclusion of assessment of sputum eosinophils, in addition to standard measures of asthma control, to guide adjustment of controller therapy in adults with moderate to severe asthma. Appraisal of the evidence regarding which adjunct controller therapy to add to ICS and at what ICS dose to begin adjunct therapy in children and adults with poor asthma control supported the 2010 CTS Consensus Summary recommendations. New recommendations for the adjustment of controller medication within written action plans are provided. Finally, priority areas for future research were identified.

CONCLUSIONS: The present document is an executive summary of the first update of the CTS Asthma Guidelines following the Canadian Respiratory Guidelines Committee's new guideline development process. Tools and strategies to support guideline implementation will be developed and the CTS will continue to regularly provide updates reflecting new evidence.

Key Words: Asthma; Clinical practice guideline; Management

\begin{abstract}
Mise à jour des lignes directrices 2012 de la Société canadienne de thoracologie : le diagnostic et la prise en charge de l'asthme chez les enfants d'âge préscolaire, les enfants et les adultes
\end{abstract}

HISTORIQUE : En 2010, la Société canadienne de thoracologie (SCT) a
publié un sommaire consensuel sur le diagnostic et la prise en charge de
l'asthme chez les enfants de six ans et plus et chez les adultes, y compris une
mise à jour du continuum de prise en charge de l'asthme. L'assemblée clinique
sur l'asthme de la SCT a entrepris un processus officiel de mise à jour des
lignes directrices de pratique clinique qui portait, dans sa première mouture,
sur des sujets controversés ou des lacunes contenus dans les lignes directrices
précédentes. MÉTHODOLOGIE : Quatre questions cliniques ont été retenues comme éléments centraux de la mise à jour des lignes directrices : le rôle des mesures non effractives de l'inflammation des voies aériennes pour rajuster la thérapie anti-inflammatoire, l'amorce d'une thérapie d'appoint à la corticothérapie par aérosol (CTA) en cas d'asthme non contrôlé, le rôle d'un seul inhalateur

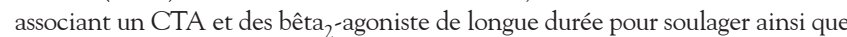
pour soulager et contrôler l'asthme et l'augmentation de la médication de contrôle en cas de perte aiguë du contrôle de l'asthme dans le cadre d'un plan de prise en charge autonome. Le groupe d'experts a respecté un processus d'adaptation pour déterminer et évaluer les lignes directrices en place sur les sujets précisés. En outre, il a procédé à des analyses bibliographiques pour trouver les analyses systématiques et les essais aléatoires et contrôlés pertinents sur le sujet. Le groupe a évalué et classé officiellement les données probantes, puis a fait 34 recommandations.

RÉSULTATS : La mise à jour des recommandations contenues dans les lignes directrices souligne qu'il y a un rôle pour l'évaluation des éosinophiles dans les expectorations, en plus des mesures standard du contrôle de l'asthme, afin d'orienter le rajustement de la thérapie de contrôle chez les adultes atteints d'asthme modéré à grave. L'évaluation des données probantes à l'égard de la thérapie de contrôle d'appoint à ajouter à la CTA et de la dose de CTA à laquelle amorcer la thérapie d'appoint chez les enfants et les adultes contrôlant mal leur asthme a étayé les recommandations du sommaire consensuel 2010 de la SCT. De nouvelles recommandations sont proposées pour rajuster la médication de contrôle dans les plans d'action écrits. Enfin, on a déterminé les domaines prioritaires des futures recherches.

CONCLUSIONS : Le présent guide de pratique clinique est un résumé de la première mise à jour des lignes directrices sur l'asthme de la SCT depuis que le comité des lignes directrices canadiennes en santé respiratoire utilise le nouveau processus d'élaboration des lignes directrices. Des outils et des stratégies pour soutenir la mise en œuvre des lignes directrices seront élaborés, et la SCT continuera de produire régulièrement des mises à jour reflétant les nouvelles données probantes.

${ }^{1}$ Queen's University, Kingston, Ontario; ${ }^{2}$ University of Montreal, Montreal, Quebec; ${ }^{3}$ University of Western Ontario, London; ${ }^{4}$ University of

Toronto, Toronto, Ontario; ${ }^{5}$ University of Alberta, Edmonton, Alberta; ${ }^{6}$ University of British Columbia, Vancouver, British Columbia;

${ }^{7}$ University of Calgary, Calgary, Alberta; ${ }^{8}$ Dalhousie University, Halifax, Nova Scotia; ${ }^{9}$ Laval University, Quebec City, Quebec

Sponsoring Organizations: Canadian Thoracic Society, and The Lung Association.

Correspondence and reprints: Canadian Thoracic Society, 300-1750 Courtwood Crescent, Ottawa, Ontario K2C 2B5. Telephone 613-569-6411,

fax 613-569-8860, e-mail ctsinfo@lung.ca 
$T_{\mathrm{sid}}^{\text {his }}$ his document presents a summary of the Canadian Thoracic Society 2012 guideline update: Diagnosis and management of asthma in preschoolers, children and adults published in Can Respir J Vol 19 No 2 March/April 2012. This clinical practice guideline (CPG) is the first formal update is the first formal update of the most recent Canadian Asthma Consensus Guidelines (1-3), the Canadian Thoracic Society (CTS) Asthma Management Continuum - 2010 Consensus Summary (4) and CTS Commentary on long-acting beta 2 -agonists (LABAs) in asthma (5), and was completed according to the Canadian Respiratory Guidelines Committee's (CRGC's) new guideline development process. The purpose is to provide evidence-based recommendations to physicians and other health care professionals for the diagnosis and management of asthma in preschoolers (under 6 years of age), children (6 to 11 years of age) and adults (12 years of age and over). We combined adults and adolescents under the heading 'adults' in the present guideline because in most instances, this grouping was a reflection of the literature that was reviewed. Where appropriate, we have separated these two groups according to age. The CTS Asthma Clinical Assembly Expert Panel will continue to identify new topics and update sections of the previous guidelines on an ongoing basis.

\section{Asthma definition}

The definition of asthma remains unchanged. Asthma is an inflammatory disorder of the airways characterized by paroxysmal or persistent symptoms such as dyspnea, chest tightness, wheezing, sputum production and cough, associated with variable airflow limitation and airway hyper-responsiveness to endogenous or exogenous stimuli (1). Inflammation and its resultant effects on airway structure and function are considered to be the main mechanisms leading to the development and maintenance of asthma.

\section{Asthma diagnosis}

Recommendations for the assessment and management of asthma addressed in the present CPG pertain to individuals with a confirmed asthma diagnosis. Asthma is diagnosed by the combination of a compatible clinical history (see asthma definition) and objective measures of lung function in individuals 6 years of age and over. Pulmonary function criteria supportive of an asthma diagnosis include: spirometry showing reversible airway obstruction, peak expiratory flow (PEF) variability, and a positive challenge test such as methacholine or exercise challenge (Table 1) $(1,4)$. In preschoolers, for whom it is not possible to routinely assess lung function, the combination of a careful clinical history (including family history and risk factors for asthma development) and physical examination are used to differentiate asthma from other causes of episodic respiratory symptoms (6).

\section{Topics in the 2012 update:}

The literature was reviewed and four main topics were critically appraised:

1. Asthma control: the role of noninvasive measures of airway inflammation (induced sputum cell counts and fraction of exhaled nitric oxide [FeNO]) to guide adjustments to therapy;

2. Adjunct controller therapy: which medication to add and, at what inhaled corticosteroid (ICS) dose;

3. ICS/LABA combination therapy used as a reliever, or as both a reliever and a controller in a single inhaler;

4. Asthma action plans: how to adjust controller therapy in the 'yellow zone'.

The panel of experts made 34 recommendations (Table 2) based on adaptation of other national and international guidelines, appraisal of the evidence from systematic reviews and published randomized controlled trials (RCTs), and input from external reviewers. Asthma control criteria (Table 3), comparative ICS dosing categories (Table 4) and the Asthma Management Continuum for individuals 6 years of age and over, and adults (Figure 1) have been updated accordingly. New recommendations regarding adjustment of controller therapy for acute loss of asthma control as part of self-management plans are presented in Table 5.

\section{Asthma control}

The primary goal of asthma management is to control the disease and, by doing so, to prevent or minimize future risk of short- and long-term complications, morbidity and mortality (4). Asthma control should be assessed regularly to guide adjustments to therapy. While airway inflammation is a fundamental feature of asthma, its assessment had not been included as a measure of asthma control until now. The CTS Asthma Assembly appraised the literature comparing the use of FeNO or sputum eosinophil count to measure airway inflammation, in addition to or instead of standard measures of asthma control to guide adjustment of controller therapy.

We conclude that there is still insufficient evidence to recommend the use of FeNO in addition to or in lieu of standard measures of asthma control. However, treating asthma according to the results of sputum cell counts is an effective strategy to reduce severe eosinophilic asthma exacerbations in adults 18 years of age and over with moderate to severe asthma. Accordingly, we recommend sputum eosinophils be considered as an additional measure of asthma control in individuals with moderate to severe asthma who are assessed in specialized centres (Table 3).

\section{Adjunct controller therapy}

ICS remain the foundation of chronic maintenance pharmacotherapy for patients with asthma in all age groups. Leukotriene receptor antagonists (LTRAs) are acceptable, second-line, daily monotherapy for children 6 years of age and over and adults. Failure to achieve acceptable asthma control on low doses of ICS should prompt re-evaluation to identify the reason. This is often due to one or more of many factors, such as erroneous diagnosis of asthma, poor inhaler device technique, poor adherence to maintenance ICS, ongoing exposure to environmental triggers and comorbidities. Controller therapy should only be escalated after reviewing and addressing these factors. The literature was reviewed to determine at what maintenance ICS dose adjunct therapy should be considered (see Table 4 for ICS dosing categories). The three options reviewed were: increasing to medium or high doses of ICS; adding a LABA; or adding an LTRA.

We conclude that no changes are necessary to the guidance provided on this topic in the most recent CTS Asthma Management Continuum - 2010 Consensus Summary for children 6 years of age and over, and adults (4), and CTS commentary on the role of LABAs (5). We reiterate the following key messages:

- LABAs should never be used alone (as monotherapy) for asthma in any age group;

- LABAs should only be used as add-on therapy to an ICS (ideally in the same inhaler device) in any age group;

- If low-dose ICS is not adequate to maintain control:

- in children 6 to 11 years of age: increase ICS to a medium dose;

- in individuals 12 years of age and over: add a LABA to the low-dose ICS, ideally in the form of a combination inhaler.

- If asthma remains uncontrolled on medium-dose ICS in children 6 to 11 years of age:

- the addition of either a LABA or LTRA are both therapeutic options, and treatment decisions should be individualized.

- If asthma remains uncontrolled on the combination of an ICS and LABA in individuals 12 years of age and over:

- consider the addition of an LTRA;

- consider referral to a specialist for assessment.

- High doses of ICS may be associated with significant side effects in children and adults, and should only be used by asthma specialists. 
TABLE 1

Diagnosis of asthma: Pulmonary function criteria

\begin{tabular}{|c|c|c|}
\hline Pulmonary function measurement & Children ( 6 years of age and over) & Adults \\
\hline \multicolumn{3}{|l|}{ Preferred: Spirometry showing reversible airway obstruction } \\
\hline $\begin{array}{l}\text { Increase in } \mathrm{FEV}_{1} \text { after a bronchodilator or after course of } \\
\text { controller therapy }\end{array}$ & $\geq 12 \%$ & $\geq 12 \%$ (and a minimum $\geq 200 \mathrm{~mL}$ ) \\
\hline \multicolumn{3}{|l|}{ Alternative: Peak expiratory flow variability } \\
\hline \multirow[t]{2}{*}{ Diurnal variation ${ }^{\dagger}$} & Not recommended & $>8 \%$ based on twice daily readings; \\
\hline & & $>20 \%$ based on multiple daily readings \\
\hline \multicolumn{3}{|l|}{ Alternative: Positive challenge test } \\
\hline a) Methacholine challenge & \multicolumn{2}{|c|}{$\begin{array}{c}\mathrm{PC}_{20}<4 \mathrm{mg} / \mathrm{mL} \\
(4-16 \mathrm{mg} / \mathrm{mL} \text { is borderline; }>16 \mathrm{mg} / \mathrm{mL} \text { is negative) }\end{array}$} \\
\hline
\end{tabular}

${ }^{*}$ Approximate lower limits of normal ratios for children and adults. ${ }^{\dagger}$ Difference between minimum am pre-bronchodilator value in 1 week and maximum pm value as $\%$ of recent maximum. FEV 1 Forced expiratory volume in $1 \mathrm{~s}$; FVC Forced vital capacity; $P C_{20}$ Provocative concentration of methacholine producing a $20 \%$ fall in FEV 1 . Reproduced from reference 4, with permission

TABLE 2

Summary of evidence-based recommendations and grading

Recommendation Description Grade

SECTION I: Noninvasive measurements of airway inflammation

$1 \mathrm{~A} \quad$ We recommend the monitoring of sputum eosinophil counts, in addition to standard measures of asthma control, to adjust

1B anti-inflammatory therapy of individuals 18 years of age and over with moderate to severe asthma in tertiary care or specialized centres.

$1 \mathrm{~B}$

We do not suggest the routine use of FeNO, either in addition to or instead of standard measures of asthma control, to adjust anti-inflammatory therapy in children or adults with asthma.

There is insufficient evidence to recommend for or against the monitoring of sputum eosinophil counts to adjust the anti-

inflammatory treatment of children and adolescents (12 to 17 years of age) with asthma.

There is insufficient evidence to make a recommendation regarding the use of FeNO either in addition to or instead of standard measures of asthma control to adjust anti-inflammatory therapy in preschoolers.

SECTION II: Adjunct therapy

General Regular need for a reliever (of any kind) merits re-evaluation to identify the reason(s) for poor asthma control. For SABA, regular Consensus use is defined as more than three doses per week.

General All treatment decisions should be based on individual characteristics which, depending on resources, could include clinical characteristics, objective measures of pulmonary function, and inflammatory markers.

General The effectiveness of each treatment decision should be carefully evaluated for its impact on current control, future risk (in particular) asthma exacerbations, and side effects.

2A We recommend initiation of adjunct therapy in adults with asthma uncontrolled despite adherence to a low dose of ICS.

2B We recommend initiation of adjunct therapy in children with asthma uncontrolled despite adherence to a medium dose of ICS.

$3 \mathrm{~A}$ In adults with asthma not achieving control despite adherence to a low dose of ICS, we recommend the addition of a LABA.

Alternative third-line options include adding an LTRA or increasing to a medium dose of ICS.

3B In children with asthma not achieving control despite adherence to a low dose of ICS, we recommend increasing to a medium dose of ICS.

3C In children not achieving asthma control on a medium dose of ICS, we suggest the addition of a LABA or LTRA.

Children who fail to achieve control on a medium dose of ICS should be referred to a specialist.

$4 \mathrm{~A} \quad$ We do not recommend the use of an ICS/LABA combination as a reliever in lieu of a FABA as a reliever in individuals 16 years of $1 \mathrm{~B}$ age and over with mild intermittent asthma on no maintenance controller therapy.

There is insufficient evidence to make a recommendation regarding the efficacy of an ICS/LABA combination as a reliever in individuals under 16 years of age on no maintenance controller therapy.

There is insufficient evidence to make a recommendation regarding the efficacy of an ICS/LABA combination as a reliever in children and adults on ICS monotherapy. 
TABLE 2 - CONTINUED

\title{
Recommendation Description
}

$5 \mathrm{~A} \quad$ We do not suggest use of a single inhaler of budesonide/formoterol as a reliever and a controller as a self-management strategy $2 \mathrm{~B}$

in lieu of ensuring adherence to low-dose ICS (400 mcg/day CFC-BDP equivalent) and a FABA as a reliever in individuals

12 years of age and over.

There is insufficient evidence to make a recommendation regarding the use of a single inhaler of budesonide/formoterol as a reliever and a controller as a self-management strategy compared with fixed-dose ICS/LABA in individuals who are exacerbation prone despite adherence to low-dose ICS.

$5 B$

We do not currently suggest the use of a single inhaler of budesonide/formoterol as a reliever and a controller as a selfmanagement strategy in children 4 to 11 years of age, and this approach is not approved in Canada in this age group. We suggest the use of a single inhaler of budesonide/formoterol as a reliever and a controller at the same ICS dose be considered in individuals 12 years of age and over with asthma uncontrolled on fixed-dose ICS/LABA combination therapy in lieu of increasing the ICS dose of the combination therapy.

$5 \mathrm{D}$

Use of a single inhaler of budesonide/formoterol as a reliever and a controller and 'guideline best practice' of a practitioner titrating controller medication according to current control are both effective therapeutic options in individuals 12 years of age and over. We suggest that the choice between these therapeutic strategies be individualized based upon patient preferences and steroid load.

SECTION IV: Controller therapy for action plans

\section{Part 1: Intermittent ICS}

$6 \mathrm{~A} \quad$ We recommend daily ICS in lieu of starting intermittent ICS at the onset of an episode of acute loss of asthma control in patients 1 with mild persistent asthma.

$6 \mathrm{~B}$

We recommend that the safest and minimal effective ICS dose be prescribed to minimize side effects in all age groups, particularly in children to address the concern regarding growth velocity.

Part 2: Escalating ICS

$7 \mathrm{~A} \quad$ We suggest that children and adults already on maintenance ICS monotherapy do not routinely double the dose of their ICS as $2 \mathrm{~B}$ part of a written action plan (or self-management plan) at the onset of an episode of acute loss of asthma control.

$7 \mathrm{~B}$

We suggest a trial of increasing the ICS maintenance dose by four- or five-fold for 7 to 14 days as part of an action plan (or self- $2 \mathrm{C}$ management plan) for acute loss of asthma control in adults with a history of severe exacerbations in the past year requiring systemic steroids.

$7 \mathrm{C}$

We recommend that children on maintenance ICS monotherapy do not routinely increase by a four-fold or more the dose of their ICS as part of a self-management plan for acute loss of asthma control. There is no evidence of efficacy and there is evidence of potential harm.

Part 3: Fixed-dose ICS/LABA combination

$8 \mathrm{~A} \quad$ There is insufficient evidence regarding the efficacy of starting a fixed dose of ICS/LABA combination with a SABA as a reliever as part of a self-management plan for children and adults on no controller therapy or on ICS monotherapy experiencing an acute loss of asthma control. Currently, we do not recommend this approach.

$8 \mathrm{~B}$

We suggest increasing budesonide/formoterol to a maximum of four inhalations twice daily (i.e. budesonide/formoterol-AMD) for 7 to14 days in individuals 16 years of age and over on maintenance fixed-dose budesonide/formoterol as part of a selfmanagement plan for acute loss of asthma control.

There is insufficient evidence to make a recommendation regarding the efficacy of budesonide/formoterol-AMD self-management strategy in individuals 12 to 15 years of age and budesonide/formoterol-AMD is not approved in Canada for use in individuals under 12 years of age.

In individuals on budesonide/formoterol, there is insufficient evidence to make a recommendation regarding budesonide/ formoterol-AMD as part of a self-management plan compared with escalation of ICS dose in any age group.

There is insufficient evidence to make a recommendation regarding the efficacy of escalating ICS strengths of ICS/LABA combinations in individuals on fixed-dose fluticasone/salmeterol or mometasone/formoterol maintenance therapy, as part of a self-management plan for acute loss of control.

$8 \mathrm{C}$

In adults who are exacerbation-prone despite maintenance fixed-dose fluticasone/salmeterol or mometasone/formoterol, we suggest either a four-fold or greater increase in ICS dose for 7 to 14 days or a course of systemic steroids be considered as part of a self-management plan for acute loss of asthma control.

Part 4: Systemic corticosteroids

9A We do not recommend routinely adding oral corticosteroids in adults as part of a written action plan at the onset of an episode of $2 \mathrm{C}$ acute loss of control.

$9 B$

$9 \mathrm{C}$

9D

$9 \mathrm{E}$

We suggest that health care practitioners add oral corticosteroids in adults with recent severe exacerbations who fail to respond to inhaled SABA therapy as part of a written action plan at the onset of an episode of acute loss of control.

Prednisone dose and duration in adults should be individualized based on previous or current response. We suggest a dose of 30 to $50 \mathrm{mg} /$ day for at least 5 days.

We do not recommend routinely adding oral corticosteroids in preschoolers and children as part of a written action plan at the onset of an episode of acute loss of control.

We suggest health care practitioners add oral corticosteroids in children with recent severe exacerbations who fail to respond to inhaled SABA as part of a written action plan at the onset of an episode of acute loss of control (GRADE 2B). Practitioners are advised to ensure that the child is adequately immunized in general, and in particular immune to, or vaccinated for, chickenpox or advise parents to seek prompt medical attention if exposed to chickenpox while taking oral steroids (Consensus).

$9 \mathrm{~F}$

Prednisone/prednisolone dose and duration in children should be individualized based on previous or current response.

We suggest a dose of $1 \mathrm{mg} / \mathrm{kg} /$ day (maximum $50 \mathrm{mg}$ ) for at least 3 days.

$9 G$

In all age groups, the use of oral corticosteroids for an episode of acute loss of asthma control requires prompt practitioner reassessment of the current episode and review of the daily controller therapy. Frequent courses of oral corticosteroid should prompt referral to a specialist.

B

\author{
(
}


$\mathrm{ICS} / \mathrm{LABA}$ as a reliever and budesonide/formoterol as a reliever and a controller

The 2003 Canadian Asthma Consensus Guidelines broadened the recommended class of reliever medication from short- to fast-acting bronchodilator (1). One LABA (formoterol [FORM]) is also a fast-acting beta $_{2}$-agonist (FABA). However, use of formoterol alone (without an ICS) as a reliever in asthma is not recommended and it is not approved for this indication in Canada because of concerns that LABAs increase the risk of asthma-related deaths. Whether concomitant use of an ICS mitigates this risk is the subject of ongoing research. Combination inhalers of controller and reliever medication offer theoretical advantages both for chronic management to maintain asthma control and as part of guided asthma self-management strategies and action plans that prompt patient-initiated adjustments of both reliever and controller therapy to treat acute loss of asthma control.

FORM in combination with budesonide (BUD) (but not in combination with mometasone) is approved in Canada for use as a reliever in individuals 12 years of age and over. Until now, the CTS had not formally reviewed the efficacy of an ICS/LABA combination agent as a reliever or the efficacy of a single inhaler of BUD/ FORM as a reliever and a controller. The literature was reviewed to examine the efficacy of: i)a single inhaler of BUD/FORM as a reliever compared with a FABA (either short-acting beta agonist [SABA] or fast-acting LABA); and ii) a single inhaler of BUD/ FORM as a reliever and a controller compared with the usual maintenance dose of controller (ICS, lower fixed-dose ICS/LABA) or 'best practice' of a practitioner adjusting maintenance therapy at office visits.

We conclude that:

- SABAs are appropriate relievers for asthma in all age groups and severity, and are the preferred class of reliever for use on demand in all patients with mild asthma (including individuals not on controller therapy and individuals on ICS monotherapy);

- BUD/FORM as a reliever may be considered in exacerbationprone individuals 12 years of age and over with moderate asthma and poor control despite fixed-dose maintenance ICS/LABA combination;

- Use of a single inhaler of BUD/FORM as a reliever and a controller may be of value in select subgroups of individuals 12 years of age and over, particularly exacerbation-prone individuals with uncontrolled asthma despite high maintenance doses of ICS or ICS/LABA combination therapy;

- Additional data are needed to clarify the risk/benefit of the use of a single inhaler of BUD/FORM as a reliever and a controller compared with conventional best practice of a practitioner adjusting maintenance therapy.

Action plans: What to do in the 'yellow zone'

Written action plans are the foundation of guided-self management. Although all major guidelines recommend the use of written action
TABLE 3

Asthma control criteria

\begin{tabular}{ll}
\hline Characteristic & Frequency or value \\
\hline Daytime symptoms & $<4$ days/week \\
Night-time symptoms & $<1$ night/week \\
Physical activity & Normal \\
Exacerbations & Mild, infrequent \\
Absence from work or school due to asthma & None \\
Need for a fast-acting beta -agonist $^{- \text {ald }}$ & $<4$ doses/week \\
FEV ${ }_{1}$ or PEF & $\geq 90 \%$ personal best \\
PEF diurnal variation & $<10 \%-15 \%$ \\
Sputum eosinophils &
\end{tabular}

*Diurnal variation is calculated as the highest peak expiratory flow (PEF) minus the lowest divided by the highest peak flow multiplied by 100 for morning and night (determined over a two-week period). ${ }^{+}$Consider in adults with uncontrolled moderate to severe asthma who are assessed in specialist centres. FEV ${ }_{1}$ Forced expiratory volume in $1 \mathrm{~s}$. Adapted from reference 4

plans, specific evidence-based recommendations regarding adjustment to controller therapy in the 'yellow zone' are either absent or unclear. The Asthma Assembly, therefore, undertook to review the literature and provide guidance regarding how to escalate controller therapy for loss of control (in the 'yellow zone'), based on the baseline maintenance ('green zone') medication. Specifically, we reviewed the evidence examining the efficacy of escalation of controller medication as part of a written action plan for preschoolers, children and adults with acute loss of asthma control in patients not on regular controller therapy, ICS monotherapy, LTRA monotherapy, or ICS and LABA combination therapy.

Recommended step-up ('yellow zone') therapy, based on maintenance ('green zone') therapy, is summarized according to age group in Table 5 . The following key messages warrant special emphasis:

- Adherence to maintenance ('green zone') therapy is a fundamental component of written action plans;

- Evidence does not support the use of intermittent ICS started only at the onset of an episode of loss of asthma control in children or adults, which underlines the importance of prescribing and ensuring adherence to daily controller therapy;

- The common practice of doubling the dose of ICS has not been shown to be efficacious in RCTs and, therefore, is not routinely recommended;

- A trial of four-fold or greater increase in ICS dose for 7 to 14 days is suggested in adults with a history of severe exacerbations in the past year. This is not recommended in preschoolers, children or adolescents;

- The most appropriate 'yellow-zone' therapy for individuals on fixed-dose ICS/LABA therapy is not yet known. There is

\section{TABLE 4}

Comparative inhaled corticosteroids (ICS) dosing categories in children and adults

\begin{tabular}{|c|c|c|c|c|c|c|c|}
\hline \multirow[b]{3}{*}{ Corticosteroid } & \multirow[b]{3}{*}{ Trade name } & \multicolumn{6}{|c|}{ Daily ICS dose, $\mathrm{mcg}$} \\
\hline & & \multicolumn{3}{|c|}{ Pediatric (6 to 11 years of age) } & \multicolumn{3}{|c|}{ Adult (12 years of age and over) } \\
\hline & & Low & Medium & High & Low & Medium & High \\
\hline Beclomethasone dipropionate HFA & $\operatorname{QVAR}^{\dagger}$ & $\leq 200$ & $201-400^{\mathrm{a}}$ & $>400^{\mathrm{a}}$ & $\leq 250$ & $251-500$ & $>500$ \\
\hline Ciclesonide $^{*}$ & Alvesco§ & $\leq 200$ & $201-400^{a}$ & $>400^{\mathrm{a}}$ & $\leq 200$ & $201-400$ & $>400$ \\
\hline Fluticasone & Flovent MDI and spacer; Flovent Diskus & $\leq 200$ & $201-400$ & $>400^{a}$ & $\leq 250$ & $251-500$ & $>500$ \\
\hline Mometasone & Asmanex Twisthaler** & & & & 200 & $\geq 400-800$ & $>800^{b}$ \\
\hline
\end{tabular}

Dosing categories are approximate, based on a combination of approximate dose equivalency as well as safety and efficacy data rather than available product formulations. *Licensed for once daily dosing in Canada (a: Daily doses of beclomethasone dipropionate HFA >200 mcg/day, ciclesonide >200 mcg/day and fluticasone $>400$ mcg/day are not approved for use in children in Canada [highlighted]); † Graceway Pharmaceuticals, Canada; ${ }^{\ddagger}$ AstraZeneca Inc, Canada; $\$$ Nycomed Canada Inc; TGlaxoSmithKline Inc, Canada; **Merck \& Co Inc, USA (b: Daily doses of mometesone >800 mg/day are not approved for use in adults in Canada [highlighted]). Adapted from reference 1 


\section{Asthma Management Continuum Children (6 years and over) and Adults}

\begin{tabular}{|l|}
\hline Regularly Reassess \\
- Control \\
- Spirometry or PEF \\
- Inhaler technique \\
- Adherence \\
- Triggers \\
- Comorbidities \\
- Sputum eosinophils§ \\
\hline
\end{tabular}

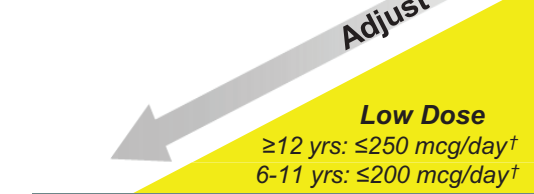

*Second-Line: Leukotriene Receptor Antagonist (LTRA)

Inhaled Corticosteroid (ICS)*

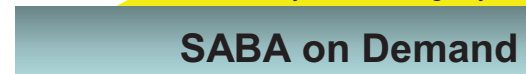

Medium Dose

$251-500 \mathrm{mcg} /$ day $^{\dagger}$ $201-400 \mathrm{mcg} /$ day $^{\dagger}$
212 yrs. Add LTRA

6-11 yrs: Add LABA or LTRA

$\geq 12$ yrs: Add LABA*

6-11 yrs: Increase ICS

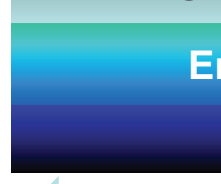

Controlled

SABA or ICS/LABA ${ }^{\ddagger \rrbracket}$ on Demand

Environmental Control, Education and Written Action Plan

\section{Confirm Diagnosis}

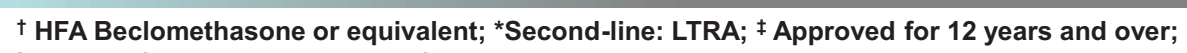

" Using a formulation approved for use as a reliever;

$\S$ In adults 18 years and over with moderate to severe asthma.

Figure 1) Management hinges on confirming the diagnosis. All individuals with confirmed asthma should receive self-management education, including a written action plan. Very mild intermittent asthma may be treated with a short-acting beta $a_{2}$-agonist (SABA) taken as needed. SABAs are recommended for relief of symptoms; individuals 12 years of age and over with moderate to severe asthma (particularly those who are exacerbation prone and have poor control) who are taking an ICS/LABA formulation approved also for use as a reliever may do so. Inhaled corticosteroids (ICS) should be introduced early as the initial maintenance treatment for asthma even in individuals who report asthma symptoms less than three times a week. LTRA are second-line monotherapy for mild asthma. If asthma is not adequately controlled by low doses of inhaled corticosteroids, additional therapy should be considered. In children 6 years of age and

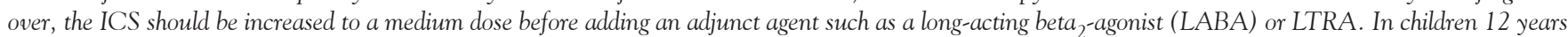
of age and over and adults, a LABA should be considered first as adjunct therapy. A LABA should only be used in combination with an ICS. Increasing to a medium dose of ICS or the addition of an LTRA are third-line therapeutic options. Theophylline may be considered as a fourth-line agent in adults. Severely uncontrolled asthma may require additional treatment with prednisone. Omalizumab may be considered in individuals 12 years of age and over with atopic asthma poorly controlled despite high doses of ICS and appropriate add-on therapy, with or without prednisone. Asthma symptom control and lung function tests, inhaler technique, adherence to asthma treatment, exposure to asthma triggers in the environment, and the presence of co-morbidities should be reassessed at each visit and before altering the maintenance therapy. Consider also assessment of sputum eosinophils in adults with uncontrolled moderate to severe asthma managed in specialized centres. After achieving acceptable asthma control for at least a few weeks to months, the medication should be reduced to the minimum necessary dose to achieve adequate asthma control and prevent future risk of exacerbations. HFA: Hydrofluoroalkane; IgE: Immunoglobulin E; mcg: Micrograms; PEF: Peak expiratory flow; yrs: Years

insufficient evidence to make a recommendation for or against escalating ICS strengths of fluticasone propionate/salmeterol or mometasone/FORM as part of self-management action plans. Older adolescents (16 years of age and over) and adults on BUD/ FORM may benefit from adjustable maintenance dose (increasing to a maximum of four inhalations twice daily);

- Oral corticosteroids (OCS) are beneficial in the management of established asthma exacerbations. In adults, most of the evidence of the efficacy of OCS arises from trials of treatment of asthma in the emergency department setting and it may not be valid to extrapolate that as justification for its inclusion in action plans. There is at least some direct evidence from RCTs of the efficacy of OCS in pediatric action plans. As part of a written action plan, in children and adults, we suggest OCS be reserved for individuals with recent severe exacerbations who fail to respond to inhaled SABAs within 6 to 8 hours.
Guideline dissemination, implementation and knowledge translation tools

The present CPG summary will be available for viewing and download from the CRGC website (www.respiratoryguidelines.ca) and the Canadian Respiratory Journal website (www.pulsus.com). The trifold pocket Asthma Management brochure ('Slim Jim') will be updated to reflect these new recommendations. It will also be available via the CRGC website and will be mailed along with the CPG to Provincial Lung Associations for distribution to local target users, such as family physicians, allied health professionals working in respiratory care and certified respiratory educators (CREs). A slide kit for teaching and self-directed learning will be posted for viewing and download on the CRGC website. The CPG and derivative resources and tools will be disseminated across Canada via CTS e-bulletins to individuals and organizations that have an interest in this topic area.

The CTS Asthma Clinical Assembly welcomes the opportunity to partner with other organizations and stakeholders in the development 
TABLE 5

Action plan recommendations based on age and maintenance controller therapy

\begin{tabular}{|c|c|c|}
\hline Maintenance therapy & Recommended reliever* & Recommended controller step-up therapy for the Action Plan 'Yellow zone' \\
\hline \multicolumn{3}{|c|}{ Preschoolers (under 6 years of age ) and children (6 to 11 years of age) } \\
\hline \multirow[t]{2}{*}{ No maintenance } & SABA & 1st choice: None \\
\hline & & 2nd choice: Consider starting regular controller therapy \\
\hline \multirow[t]{2}{*}{ ICS } & SABA & 1st choice: None \\
\hline & & 2nd choice: Prednisone/prednisolone $1 \mathrm{mg} / \mathrm{kg} \times 3-5$ days $§$ \\
\hline \multirow[t]{2}{*}{ ICS/LABA $\pi$} & SABA & 1st choice: None \\
\hline & & 2nd choice: Prednisone/prednisolone $1 \mathrm{mg} / \mathrm{kg} \times 3-5$ days ${ }^{\S}$ \\
\hline \multicolumn{3}{|c|}{ Adults (12 years of age and over) } \\
\hline \multirow[t]{2}{*}{ No maintenance } & SABA & 1st choice: None \\
\hline & & 2nd choice: Consider starting regular controller therapy \\
\hline \multirow[t]{2}{*}{ ICS } & SABA & 1st choice: Trial of $\geq 4$-fold increase in ICS for $7-14$ days $^{\dagger}$ \\
\hline & & 2nd choice: Prednisone $30 \mathrm{mg}$ to $50 \mathrm{mg}$ for at least 5 days $^{\dagger}$ \\
\hline \multicolumn{3}{|l|}{ ICS/LABA } \\
\hline \multirow[t]{2}{*}{ BUD/FORM } & SABA OR BUD/FORM & $\begin{array}{l}\text { 1st choice: Increase BUD/FORM to maximum of } 4 \text { inhalations twice daily for } 7-14 \text { days OR BUD/ } \\
\text { FORM as a reliever and a controller (maximum } 8 \text { inhalations/day) }\end{array}$ \\
\hline & & 2nd choice: Prednisone $30 \mathrm{mg}$ to $50 \mathrm{mg}$ for at least 5 days \\
\hline \multirow[t]{3}{*}{ FP/SALM or MOM/FORM } & 1st choice: SABA & 1st choice: Trial of $\geq 4$-fold increase in ICS (higher ICS strength of ICS/LABA combination or extra ICS) \\
\hline & 2nd choice: BUD/FORM ${ }^{\ddagger}$ & for $7-14$ days $^{\dagger}$ \\
\hline & & 2nd choice: Prednisone $30-50 \mathrm{mg}$ for at least 5 days $^{\dagger}$ \\
\hline
\end{tabular}

*If regular need for reliever or frequent step-up therapy, identify reason for poor control, adjust (start or increase) maintenance therapy; ${ }^{\prime}$ In individuals $\geq 15$ years of age with a history of severe acute loss of asthma control in the preceding year; ${ }^{\ddagger}$ In exacerbation-prone individuals; §In children with a recent history of severe exacerbation and suboptimal response to SABA during index exacerbation; "Does not apply to preschoolers. BUD/FORM Budesonide/formoterol; FP/SALM Fluticasone propionate/ salmeterol; ICS Inhaled corticosteroid; LABA Long-acting beta ${ }_{2}$-agonist; max Maximum; MOM/FORM Mometasone/formoterol; SABA Short-acting beta ${ }_{2}$-agonist

of educational resources and tools that support implementation and uptake of the guidelines with various target groups. The CTS Asthma Clinical Assembly will continue its collaboration with the Ontario Lung Association's Provider Education Program (www.olapep.ca) to update the asthma e-Learning modules and cases; an 'Action Plan' module is in development. The Assembly will continue to collaborate with the Lung Association's RESPTrec ${ }^{\circledR}$ Program to update the asthma curriculum for CREs (www.resptrec.org/). The CTS Asthma Clinical Assembly will also work with the Canadian Lung Association (CLA) on The Pan-Canadian REspiratory STandards INitiative for Electronic Health Records (PRESTINE) initiative (7), to recommend asthma elements that prompt and enable adherence with the CTS Asthma Guidelines.

The CTS Asthma Clinical Assembly Expert Panel recognizes and acknowledges that there may be resource implications related to implementing the present CPG. These were taken into consideration in forming the recommendations (eg, limiting the recommendation that induced sputum be accessible in specialized centres). It is hoped that the implementation of these recommendations will be cost-neutral or cost-effective by promoting evidence-based diagnosis, assessment and use of medications.

Monitoring adherence with guideline recommendations

The following parameters may be used to monitor or audit adherence with some of the key recommendations contained in the present CPG:

- asthma diagnosis is confirmed by an objective measure of lung function in individuals 6 years of age and over;

- asthma control criteria are assessed at asthma follow-up visits (including sputum eosinophils in individuals 18 years of age and over with moderate asthma in tertiary care or specialized centres);

- patient has a reliever (SABA for any severity; or BUD/FORM for exacerbation-prone individuals 12 years of age and over with moderate asthma and poor control);

- patient is prescribed a controller (ICS or LTRA, or ICS/LABA, or prednisone);

- adjunct controller therapy (either a LABA or LTRA) is prescribed for children with asthma uncontrolled on a medium dose of ICS;

- adjunct controller therapy (LABA as an ICS/LABA combination) is prescribed for adults with asthma uncontrolled on a low dose of ICS;

- patient/parent/caregiver receives self-management education, including a written asthma action plan (with 'yellow zone' instructions based upon maintenance 'green zone' controller therapy); and

- children with asthma uncontrolled on a medium dose of ICS are referred to an asthma specialist.

EDITORIAL INDEPENDENCE: The CTS Asthma Clinical Assembly is accountable to the CTS Respiratory Guidelines Committee and the CTS Board of Directors. The CTS Asthma Clinical Assembly is functionally and editorially independent from any funding sources of the CTS and does not receive any direct funding from external sources. The CTS receives unrestricted grants, which are combined into a central operating account to facilitate the knowledge translation activities of CTS Clinical Assemblies. Sources include the Canadian Institutes of Health Research, AstraZeneca Canada, Boehringer Ingelheim Canada, GlaxoSmithKline Inc, Pfizer and Talecris. No funders played a role in the collection, review, analysis or interpretation of the scientific literature or in any decisions regarding the recommendations or key messages presented in this document.

DISCLOSURE OF COMPETING INTERESTS: Members of the Asthma Clinical Assembly declared potential conflicts of interest at the time of appointment and these were updated throughout the process in accordance with the CTS Conflict of Interest Disclosure Policy. Individual member conflict of interest statements are posted at http://www.respiratoryguidelines.ca/guideline/asthma.

ACKNOWLEDGEMENTS: The authors thank: Canadian Lung Association staff Janet Sutherland and Anne Van Dam for outstanding administrative support; Candice Brown, Stella Muthuri and Kristen Curren for dedicated project management; Karen Spithoff for methodological expertise; and the Canadian Respiratory Guidelines Committee for expert support and guidance. The authors also thank the expert peer reviewers: Dr. Alan Bell (Toronto, Ontario), Dr. Robert Cowie (Calgary, Alberta), Dr. Harold Kim (Kitchener, Ontario), for the Canadian Society of Allergy and Clinical Immunology), Jan Neumann, CRE (Saskatoon, Saskatchewan), Dr. Mary Noseworthy (Calgary, for the CTS Pediatric Clinical Assembly), Dr. Francisco Noya (Montreal, Quebec), for the Canadian Society of Allergy and Clinical Immunology. 


\section{REFERENCES}

1. Lemiere C, Bai T, Balter M, et al. Adult Asthma Consensus Guidelines Update 2003. Can Respir J 2004;11(Suppl A):9A-18A.

2. Becker A, Berube D, Chad Z, et al. Canadian Pediatric Asthma Consensus guidelines, 2003 (updated to December 2004): introduction. CMAJ 2005;173(6 Suppl):S12-S4.

3. Becker A, Lemiere C, Berube D, et al. Summary of recommendations from the Canadian asthma consensus guidelines, 2003. CMAJ 2005;173(6 Suppl):S3-11.

4. Lougheed MD, Lemiere C, Dell SD, et al. Canadian Thoracic Society Asthma Management Continuum - 2010 Consensus Summary for children six years of age and over, and adults. Can Respir J 2010;17:15-24.
5. Lougheed MD, Lemiere C, Dell S, et al. Canadian Thoracic Society Asthma Committee commentary on long-acting beta-2 agonist use for asthma in Canada. Can Respir J 2010;17:57-60.

6. Global Strategy for the Diagnosis and Management of Asthma in Children 5 Years and Younger. Global Initiative for Asthma (GINA), 2009. <www.ginasthma.org>

7. Lougheed MD, Minard JP, Dworkin S, et al.

The Pan-Canadain REspiratory STandards INiative for Electronic Health Records (PRESTINE) Initiative: Proceedings of the 2011 National Forum. Can Respir J 2012;19:117-126. 


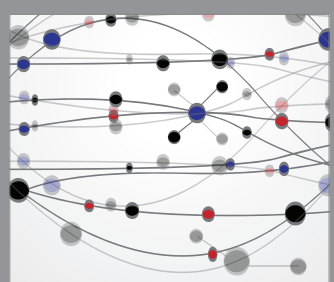

The Scientific World Journal
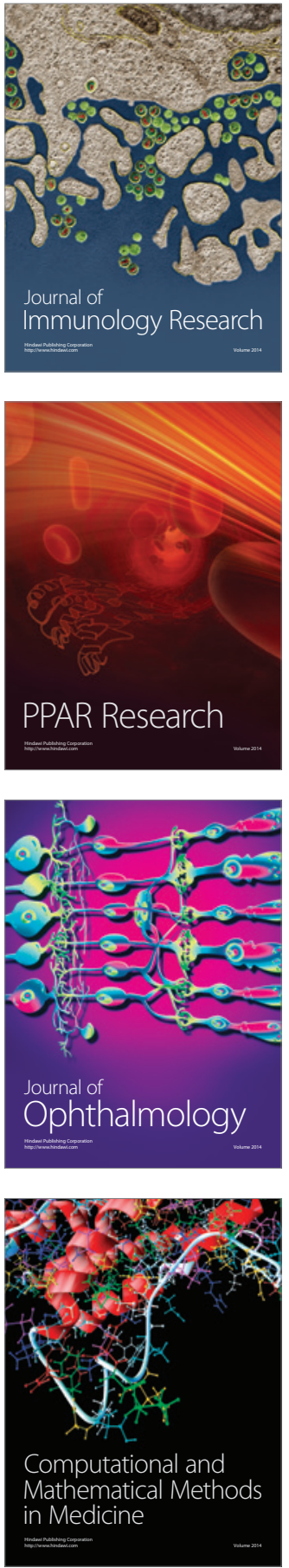

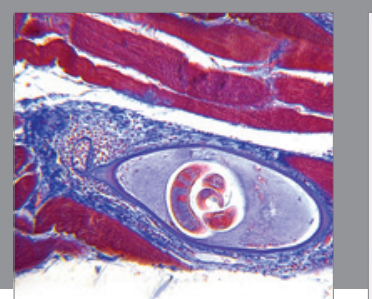

Gastroenterology Research and Practice

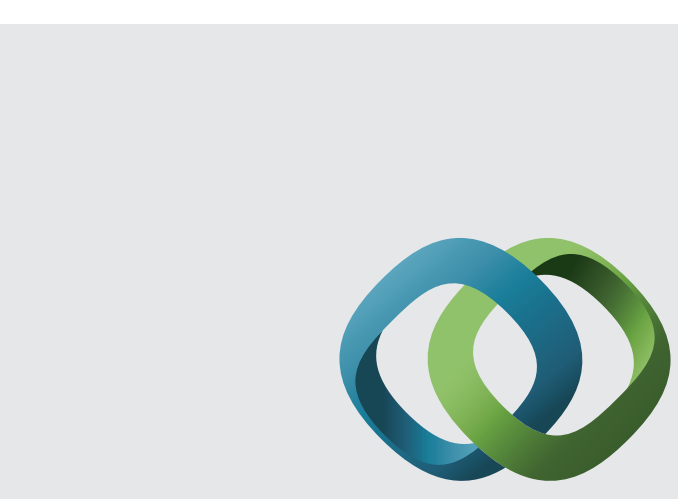

\section{Hindawi}

Submit your manuscripts at

http://www.hindawi.com
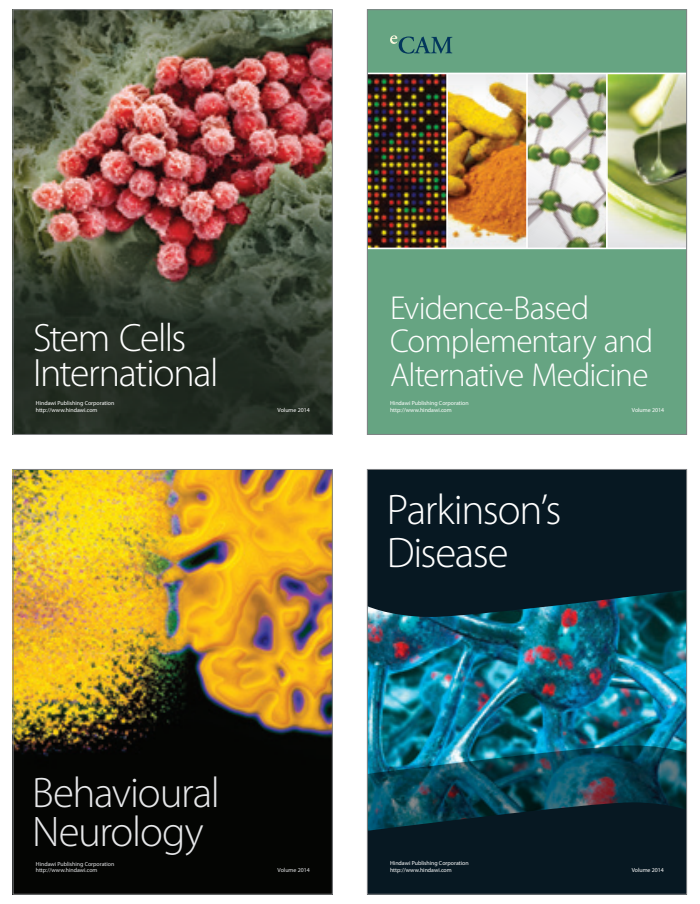
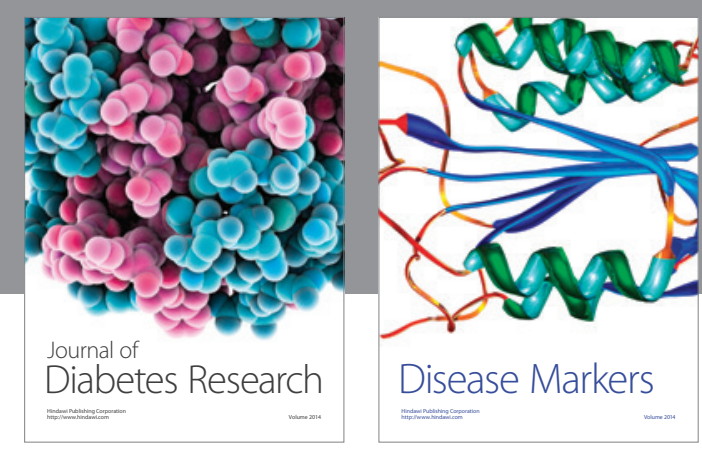

Disease Markers
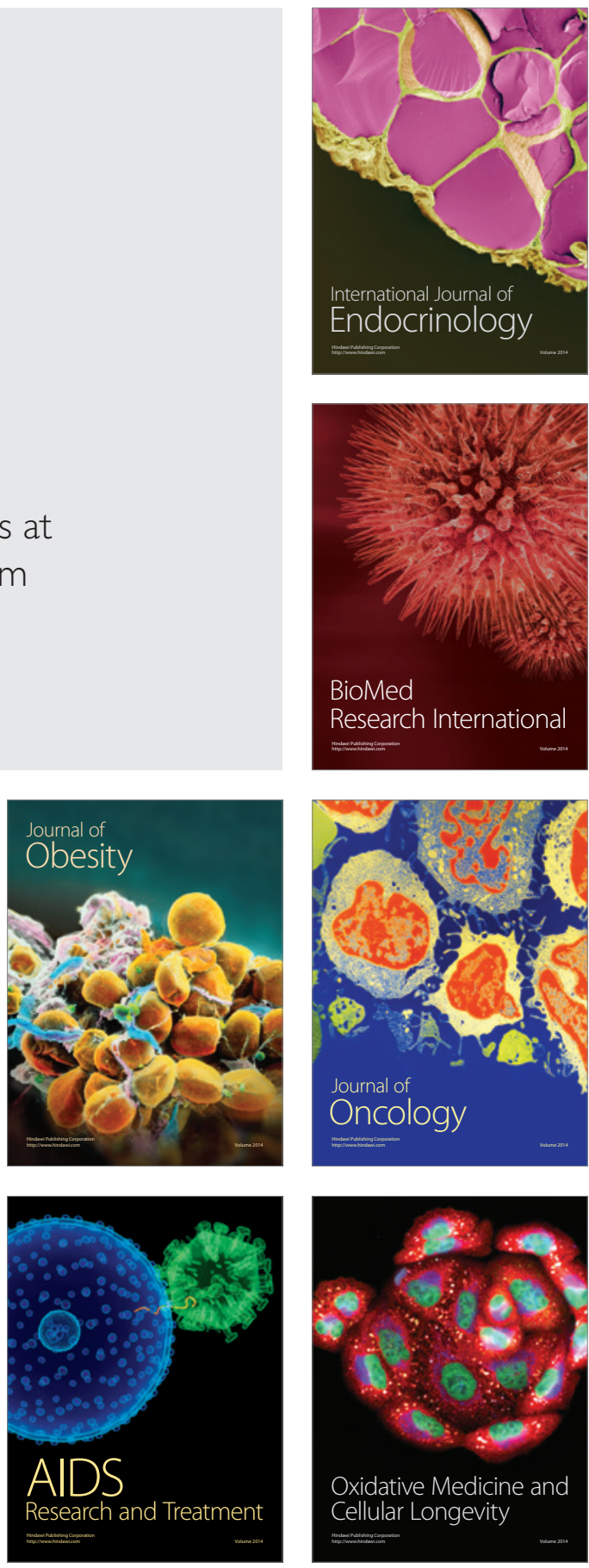